\title{
Controversy
}

\section{Ventilator settings for newborn infants}

\author{
C A RAMSDEN AND E O R REYNOLDS
}

Department of Paediatrics, University College London

The selection of appropriate mechanical ventilator settings for newborn infants is a controversial topic. The purpose of this review is to summarise the origins of the controversy and then to concentrate on the interaction between ventilator variables and the pathophysiology of the lung. As lung mechanics differ widely in different diseases we argue that careful consideration must be given to the mechanical properties of the lung when deciding on a suitable ventilator regimen for a particular infant.

\section{Origin of early recommendations}

Many years ago, one of us made recommendations for the ventilation of infants with severe hyaline membrane disease. These recommendations are still followed by some, but others claim that they promote pneumothoraces and that better results are obtained if different settings are used-notably, faster respiratory rates. If the current debate about how best to set ventilators is to be clarified the origin of the early recommendations must be understood.

Mechanical ventilation for newborn infants with respiratory failure became widespread in the late 1960 s and early 1970 s. Although it was immediately clear that the lives of preterm infants with recurrent attacks of apnoea and relatively normal lungs could be saved, great difficulty was encountered in infants with severe hyaline membrane disease (HMD).

The conventional way of setting the ventilator at that time was to select a rate of $60-80$ breaths per minute for infants with HMD and if possible to synchronise the machine with the infant's own breathing pattern. At these rates the arterial carbon dioxide tension could usually be easily controlled but the oxygen tension could not, unless very high peak airway pressures (often $35-50 \mathrm{~cm} \mathrm{H}_{2} \mathrm{O}$ ) were used. ${ }^{1}$ The vast majority of the infants died, either rapidly of hypoxaemia or more slowly (at about 2 weeks of age) from an aggressive form of lung fibrosis, which was termed bronchopulmonary dysplasia. ${ }^{23}$ Evidence was obtained from observations of ventilator variables and autopsy findings that the main cause of bronchopulmonary dysplasia at that time was distension and disruption of the terminal airways caused by the use of very high peak airway pressures. ${ }^{34}$

Experiments were therefore performed to see if ways could be found to ventilate babies with severe HMD at lower peak airway pressures. ${ }^{56}$ Modified Bennett PR2 ventilators (time cycled, pressure limited, intermittent flow machines) were used, set to provide a plateau of peak airway pressure during inspiration. The major findings of these studies were that if a rather slow rate was selected (30 breaths/ minute) arterial oxygen tension was higher than at a fast rate (an observation also made by Smith $e t a l^{7}$ ) and that the oxygen tension could generally be raised further by manoeuvres that increased mean airway pressure, notably by increasing inspiration: expiration (I:E) ratio. Arterial carbon dioxide tension could most readily be manipulated by small changes in ventilator rate or in the difference between peak and end expiratory pressures (which altered alveolar ventilation).

These results were easy to relate to the pathophysiology of HMD. As the surfactant deficient lung is both poorly compliant and unstable, it is difficult to inflate and collapses readily during expiration. Atelectasis causing right to left shunting of blood through both intrapulmonary and extrapulmonary channels is responsible for -most of the deficit of oxygen uptake. ${ }^{8}$ Hence improvement of arterial oxygen tension was to be expected when peak airway pressure, I:E ratio, or positive end expiratory pressure were increased-the first strategy opening up collapsed lung units, the second holding them open for a greater part of each breath, and the third retarding alveolar collapse during expiration. The effect of slowing the ventilator rate in improving oxygen tension, though more complex, may in part have reflected the need for time as well as pressure for collapsed lung units to reinflate, because viscous and inertial forces must be overcome. ${ }^{49}$ Any strategy that increases the duration of the inspiratory phase, such as decreasing rate or increasing I:E ratio 
(while maintaining other variables constant) should, within limits, promote alveolar inflation, reduce right to left shunt, and improve oxygenation.

These observations on the effects of different ventilator settings led to recommendations for the management of infants with severe HMD that were designed to avoid the use of very high peak airway pressures $\left(>25 \mathrm{~cm} \mathrm{H}_{2} \mathrm{O}\right)$. This regimen was characterised by slow ventilator rates $(30-40 / \mathrm{min})$ and the use, when required, of prolonged inspiratory times ( $\mathrm{I}: \mathrm{E}$ ratio $\geqslant 1: 1)$ as a means for maintaining a satisfactory arterial oxygen tension. ${ }^{10-12}$ To keep peak airway pressure and $\mathrm{I}: \mathrm{E}$ ratio as low as possible, very high inspired oxygen concentrations were often used. As soon as recovery started the ventilator pressures and inspired oxygen concentrations were both reduced. The introduction of this regimen at University College Hospital (UCH) was associated with an immediate large increase in survival that seemed to be attributable to a reduction in deaths from 'aggressive' bronchopulmonary dysplasia. ${ }^{13}$

Many reservations must be entertained when applying this regimen today. The experiments on which it was based were performed with an intermittent flow ventilator on infants who were selected for study because they had severe HMD; hence the regimen was recommended only for similar infants. Also the observations on survival predated the regular use of continuous positive airway pressure and positive end expiratory pressure. Perhaps the greatest change though has been in the population itself; the thresholds for mechanical ventilation have become much less stringent and extremely preterm infants form a far greater proportion of the population receiving ventilation. Nevertheless, it is interesting that at $\mathrm{UCH}$, where we have used this regimen routinely for severe HMD since 1970 , the change in population does not seem to have been associated with any appreciable change in the incidence of pneumothorax - the complication currently under most debate. Between 1979 and 1983 the incidence was $21 \%$ in 88 inborn infants with gestation of 24 to 32 weeks who were ventilated for HMD. (Ramsden CA, Stewart AL. Unpublished data.)

\section{The controversy}

Some authors have experienced high incidences of pneumothorax - up to $50 \%$-in infants ventilated with slow rates and long inspiratory times, ${ }^{14}{ }^{15}$ and several publications have implied that the use of faster rates and shorter inspiratory times is associated with better results, including fewer cases of pneumothorax. ${ }^{14-22}$

Three studies have been particularly influential in popularising this view, but each suffers from problems of experimental design. ${ }^{16-18}$ Bland et al reported only two cases of pneumothorax in 24 infants ventilated for HMD at $60-110 / \mathrm{min}$ but had no control group. ${ }^{16}$ Spahr et al found a trend towards fewer air leaks in infants with HMD ventilated with I:E ratios of 1:2 than in infants ventilated with ratios of $2: 1$, but no account was taken of the severity of the disease-infants were ventilated for an average of 80 hours with an I:E ratio of 2:1, even though an inspired oxygen concentration of less than $50 \%$ was required for $60 \%$ of that time. ${ }^{17}$ Heicher and her colleagues reported that in a consecutive series of 102 infants requiring ventilation for a variety of respiratory disorders pneumothoraces developed in only $14 \%$ of those ventilated at $60 / \mathrm{min}$, significantly less than the $35 \%$ incidence in infants ventilated at $20-40 /$ min $^{18}$ The maximum peak airway pressure allowed in infants ventilated at the fast rate, however, was $30 \mathrm{~cm} \mathrm{H} \mathrm{H}_{2} \mathrm{O}$ compared with $40 \mathrm{~cm} \mathrm{H}_{2} \mathrm{O}$ for the slower rate. More recently, Pohlandt et al have reported the preliminary results of a large multicentre trial comparing slow rates and long inspiratory times with fast rates and short inspiratory times. ${ }^{19}$ A lower incidence of pneumothorax was found when fast rates were used, but no account was taken of the diagnoses.

These studies illustrate very well two fundamental problems of trial design that lie at the heart of the present debate about ventilator settings. The first is lack of appreciation that the regimen summarised earlier was developed specifically for infants with severe HMD. The use of slow rates and long inspiratory times in most other respiratory disorders or in mild or recovering HMD where the lung is fairly compliant and stable is certain to cause severe hyperinflation with disastrous results-notably, lung rupture and obstruction of the circulation. ${ }^{12}$ The second and closely related problem is the enrolment of study groups unselected either by respiratory diagnosis or by the severity of the illness.

We believe that before further trials are undertaken it is vital that consideration is given in their design to the relation between ventilator variables and lung pathophysiology, the most crucial aspect of which is the relation between the expiratory time of the ventilator and the expiratory time constant of the respiratory system.

\section{Time constant of the respiratory system}

During conventional mechanical ventilation lung deflation usually occurs passively. As the expiratory valve of the ventilator opens gas flows from the infant's lungs into the ventilator circuit propelled by the pressure gradient between the alveolar lumen 
and airway, which is generated by the elastic recoil of the lungs and chest wall.

The time taken for lung deflation depends on the magnitude of the elastic recoil (the inverse of compliance) and on the resistance of the airways. Decreased compliance shortens the time taken and increased airway resistance lengthens it.

The time required for expiration is therefore directly related to compliance and resistance and can be expressed by the following equation:

$$
\frac{\mathrm{Vt}}{\mathrm{Vo}}=\mathrm{e}^{-\frac{\mathrm{t}}{\mathrm{RC}}}
$$

where $\mathrm{Vt}$ is the volume remaining in the lung at time $t$ after the onset of expiration, Vo is the total volume exhaled when expiration is complete, and $\mathrm{e}$ is a constant with the value 2.7183 . $\mathrm{R}$ is airway resistance and $C$ is compliance of the lungs and chest wall (see Appendix for derivation of this relation).

The product of compliance and resistance is often referred to as the time constant of the respiratory system $\left(\tau_{\mathrm{RS}}\right)$ and is measured in units of time (seconds). $\tau_{\mathrm{RS}}$ provides an index of the time necessary for deflation to occur, and its relevance to ventilation becomes apparent if we solve the above equation for various durations of expiration. For example, when the duration of expiration ' $t$ ' equals $\tau_{\mathrm{RS}}$ then:

$$
\frac{\mathrm{Vt}}{\mathrm{Vo}}=\mathrm{e}^{-\frac{\mathrm{t}}{\tau_{\mathrm{RS}}}} \text { or } \frac{\mathrm{Vt}}{\mathrm{Vo}}=2 \cdot 7183^{-1}=0 \cdot 37 \text {. }
$$

Thus after the passage of a single time constant $37 \%$ of the tidal volume remains to be expired. Similarly, we can calculate that after two, three, four, or five time constants have passed the proportion remaining is $13 \cdot 5,5 \cdot 0,1 \cdot 8$, and $0 \cdot 7 \%$, respectively.

The value of $\tau_{R S}$ sets a limit to the minimum expiratory time that can be used without causing gas trapping. For practical purposes, a time equivalent to at least three time constants must be allowed if expiration is to be reasonably $(95 \%)$ complete.

\section{Time constant and disease state}

As $\tau_{\mathrm{RS}}$ is determined by the compliance and resistance of the respiratory system it varies widely according to the nature and severity of the respiratory illness.

If we use published values of $R$ and $C^{23-26}$ we can estimate the expected value of $\tau_{R S}$ for various respiratory disorders. It may be as short as 0.05 seconds in HMD (compliance $\sim 1 \mathrm{ml} / \mathrm{cm} \mathrm{H}_{2} \mathrm{O}$, resistance $\left.\sim 50 \mathrm{~cm} \mathrm{H} \mathrm{H}_{2} \mathrm{O} / \mathrm{l} / \mathrm{sec}\right)$, as long as 0.25 seconds in infants with normal lungs (compliance $5 \mathrm{ml} / \mathrm{cm} \mathrm{H} \mathrm{H}_{2} \mathrm{O}$, resistance $\sim 50 \mathrm{~cm} \mathrm{H} \mathrm{O} / \mathrm{l} / \mathrm{sec}$ ), and considerably longer in those with airways obstruction. For example, Grunstein et al recently esti- mated mean $\tau_{\mathrm{RS}}$ to be 0.5 seconds in infants with chronic lung disease. ${ }^{26}$ The time that must be allowed for expiration to occur during mechanical ventilation is therefore crucially dependent on the disease from which the infant suffers, varying more than fivefold between different diseases.

\section{Positive end expiratory pressure (PEEP) and 'inadvertent PEEP'}

If a ventilator expiratory time of less than $3 \times \tau_{R S}$ is used-for example, $<0.75 \mathrm{sec}$ for normal lungssubstantial gas trapping will inevitably occur during expiration. The effect of this is quite similar to the effect of applying PEEP and is often referred to as 'inadvertent PEEP'. Either measure will hold lung volume above the relaxed functional residual capacity - the functional residual capacity at zero inflation pressure-and may improve oxygenation if the relaxed capacity is abnormally low.

Despite this potentially beneficial effect (which newborn infants apparently set out to achieve for themselves during spontaneous breathing. See below.), we believe that inadvertent PEEP represents the major danger of employing fast rates during mechanical ventilation. Inadvertent PEEP is additive to the applied PEEP and carries no theoretical advantage over increasing the level of applied PEEP. Unlike applied PEEP, its magnitude can only be measured by sophisticated techniques, and it may be quite considerable: Simbruner and his colleagues have recently shown levels of inadvertent PEEP as high as $4-7 \mathrm{~cm} \mathrm{H}_{2} \mathrm{O}$ in infants ventilated for a variety of respiratory disorders at $30-40 / \mathrm{min}$ and with expiratory times of not less than 0.8 seconds. ${ }^{27}$ Furthermore, because the value of $\tau_{R S}$ is not static throughout the course of a respiratory illness, the level of inadvertent PEEP will vary considerably, while ventilator settings remain unchanged. During the recovery phase of HMD, for example, the use of a fast rate at a time when the $\tau_{R S}$ is increasing rapidly may cause a dramatic rise in inadvertent PEEP with the risk of carbon dioxide retention, pneumothorax, and compression of the pulmonary circulation. ${ }^{12}$

\section{Expiratory time}

Although it is tempting to use published values of $\tau_{R S}$ to estimate the minimum expiratory time that can be used in various respiratory disorders without causing air trapping, considerable caution has to be exercised as several factors may alter the effective value of $\tau_{R S}$. For example, $\tau_{R S}$ is increased by the additional resistance of the endotracheal tube, which is both variable (dependent on tube diameter 
and design) and unpredictable (due to the accumulation of secretions). ${ }^{28} 29$ The presence of PEEP (inadvertent or applied), on the other hand, may shorten the time constant by decreasing resistance (splinting airways open) and decreasing compliance (by moving the tidal flow on to a higher and flatter part of the pressure-volume curve). The mechanical behaviour of the ventilator must also be considered. In particular, the exhalation valve takes a finite time to open, during which resistance is high and expiration is slowed: the opening time is only 0.04-0.07 seconds with the Sechrist or Bear Cub BP 2001 machines but can be as much as 0.28 seconds with the Baby Bird. ${ }^{30}$

Despite these reservations, it is sobering to make some rough calculations. The value of $\tau_{R S}$ in an intubated infant with normal lungs is about 0.25 seconds. Ventilated at 60 breaths/min with an I:E ratio of $1: 1$, almost $15 \%$ of the tidal volume will be trapped in expiration; at $100 / \mathrm{min}$ the proportion increases to $30 \%$. The presence of secretions in the endotracheal tube can double respiratory system resistance ${ }^{28}$ in our last example this would increase the trapped proportion to $55 \%$. Under such circumstances inadvertent PEEP would be very great. The crucial effect of lung disease on expiratory time is evident when similar calculations are performed for infants with respiratory illnesses, using values of $\tau_{R S}$ given earlier. An immediate visual meaning to $\tau_{\mathrm{RS}}$ (as well as useful diagnostic information) is given by watching the rate of expiration in an ill infant: the lungs of an infant with severe HMD seem to collapse very rapidly, whereas those of an infant with meconium aspiration may seem to be almost fixed in inspiration.

\section{Inspiratory time}

Similar principles can be applied when considering the effect of manipulating ventilator inspiratory time. The factors affecting lung inflation are, however, more complex than for deflation and such an approach is rather crude. Nevertheless, two issues are quite important.

The first is that airway resistance is lower during inspiration than during expiration. Hence the inspiratory time necessary for lung inflation to be complete is considerably less than the corresponding expiratory time. During spontaneous breathing this effect is probably quite small, expiratory resistance being only $15-20 \%$ greater than the inspiratory resistance. Perez Fontan and his colleagues have recently reported, however, that during mechanical ventilation the combined mean expiratory resistance (respiratory system plus endotracheal tube) may be as much as 4.5 times greater than the mean inspiratory resistance. ${ }^{30}$ This effect may be explained in part by airways being splinted open by the applied inspiratory pressure. Such a striking difference between inspiratory and expiratory resistance favours gas trapping when the ventilator rate is increased; lung inflation will be complete with inspiratory times that are considerably less than the minimum effective expiratory time.

The second issue is that where atelectasis is present, as in HMD, inspiratory times considerably longer than those predicted from $\tau_{R S}$ may be required to overcome the forces that resist reexpansion of collapsed lung units (as discussed above).

\section{Inhomogeneity of the lung}

So far we have assumed that the lung behaves as if it was completely homogeneous - each lung unit operating in parallel, with an identical $\tau_{R S}$. For many practical purposes this assumption seems warranted as a linear relation between volume and flow, implying a single value for $\tau_{R S}$ (see Appendix), has been found in normal infants and infants with HMD. ${ }^{24}{ }^{25}$ As ventilatory rate increases, however, progressive inhomogeneity of $\tau_{R S}$ becomes apparent, even in normal lungs, as shown by Helliesen $e t$ $a l^{31}$ and Olinsky et al. ${ }^{32}$ There are two important consequences. Firstly, as inspiratory time shortens lung units with relatively long values for $\tau_{R S}$ become less ventilated, leading to ventilation-perfusion imbalance, and secondly, as expiratory time shortens gas trapping develops in units with long values for $\tau_{\mathrm{RS}}$.

Gross inhomogeneity of the lung is present in certain disease states-notably, meconium aspiration, which causes widespread uneven airways obstruction-and in chronic lung disease, where both resistance and compliance vary widely throughout the lung, some areas being fibrotic and others emphysematous. Clearly, no single value of $\tau_{R S}$ can be assumed when considering how best to ventilate these infants.

For infants with severe meconium aspiration, it has been suggested that advantage can be taken of the inhomogeneity of $\tau_{\mathrm{RS}}{ }^{12}$ If a very short inspiratory time is used lung units with fairly short values for $\tau_{\mathrm{RS}}$ - that is, the relatively less obstructed areas of lung-will be preferentially ventilated, thus avoiding, partly at least, the dangerous complication of severe gas trapping distal to the obstructions.

\section{Spontaneous breathing}

Infants often continue to make active respiratory efforts during mechanical ventilation, and several 
studies suggest that this activity increases the risk of pneumothorax ${ }^{33}$ and cerebral haemorrhage. ${ }^{34} \mathrm{~A}$ particular pattern of interaction between infant and ventilator, so called 'active expiration', has recently been implicated as responsible,,$^{33}$ though the mechanism by which this interaction causes pneumothorax remains uncertain. ${ }^{35}$ In a population of infants with this behaviour Greenough et al found that muscle relaxation with pancuronium almost completely prevented pneumothorax (one case in 11 infants), whereas all 11 comparable controls developed this complication. ${ }^{33}$

It is often suggested that faster ventilator rates promote 'synchrony' between infant and ventilator. While this may be a common clinical impression, surprisingly little experimental evidence is available to confirm it. Both Greenough et al and Field et al have shown some increase in the time spent in apnoea or synchronous breathing when fast ventilator rates were employed, but neither group attempted to keep the major variable affecting respiratory drive constant-namely, the carbon dioxide tension. ${ }^{20-22}$ Intuitively, it may seem reasonable to attempt to match ventilator timing to the infant's own spontaneous ventilatory cycle. Given that the control of breathing, however, depends on complex interactions between reflex responses-for example, Hering-Breuer reflex, carbon dioxide tension, sleep state, and behavioural factors - there may be a temptation to expect no more than transitory success.

There is increasing evidence that the spontaneously breathing infant (like the mouse ${ }^{37}$ ) uses a rapid respiratory rate as a means of preventing airway closure in expiration. ${ }^{36}$ By employing a rapid rate and a very short expiratory time (considerably less than $3 \times \tau_{\mathrm{RS}}$ ), complete expiration is prevented, a larger functional residual capacity is maintained, and oxygenation is improved. Attempts to achieve the same effect with a mechanical ventilator, as discussed above under 'inadvertent PEEP', are fraught with danger. Both the applied force (inadvertent PEEP) and its desired effect (raised functional residual capacity) are difficult to measure. The situation is quite unlike that of the spontaneously breathing infant whose lungs are liberally endowed with stretch receptors providing the feedback information necessary to modulate respiratory timing in accordance with the changing lung mechanics.

\section{Conclusions}

The arguments and calculations presented here are no more than illustrative, but we suggest that they show the difficulties of selecting appropriate ventilator settings for newborn infants and the folly of performing clinical trials that compare ventilator regimens in mixed populations of infants. The results of these trials must depend as much on the characteristics of the population as on the treatment applied: important beneficial effects in infants with one type of illness may be completely submerged by adverse effects in another. We contend that only far more carefully designed and disease specific trials will provide any useful answers to questions about what type of ventilation to use or how best to set the ventilator variables. Wherever possible, measurements of lung mechanics, including $\tau_{R S}$, as well as blood gases, should be employed in these trials so that the true effects of ventilation can be assessed.

Our own practice, pending further information, is based on the principles outlined above. We decide on what pattern of ventilation to use according to the respiratory problem from which the infant suffers; in so doing we hope to obtain satisfactory blood gases by tailoring the ventilator settings to suit the infant's lung mechanics. In general, we use the same disease specific guidelines as described in detail in $1979 .{ }^{12}$ We rarely use rates greater than $40 /$ min. We watch the infant's chest movements carefully to be sure that the lung is inflating and to assess whether expiration seems complete before the next ventilator breath: a visual clue about the duration of $\tau_{R S}$ can thus be obtained and the risks of inadvertent PEEP and gas trapping minimised. We often use pancuronium for muscle relaxation in the larger infants but are wary of it in the smallest ones. ${ }^{38}$

One important issue not touched on here is the potential place of ventilation of newborn infants by high frequency oscillation: ${ }^{39}$ it is at present too early to draw firm conclusions about this very promising technique.

\section{Appendix}

According to the equation of motion of the respiratory system

$$
\mathrm{P}=\frac{\mathrm{V}}{\mathrm{C}}+\mathrm{R} \dot{\mathrm{V}}
$$

where $\mathrm{P}$ is the distending pressure (airway pressure during intermittent positive pressure ventilation), C the compliance, and $\mathrm{R}$ the resistance of the lungs and chest wall. $V$ represents the volume above functional residual capacity and $\dot{V}$ the rate of gas flow measured at the airway opening.

At the onset of a passive expiration $P$ falls to zero and equation (1) can be rearranged to give

$$
\begin{gathered}
\frac{\mathrm{V}}{\dot{\mathrm{V}}}=-\mathrm{RC} \\
\text { or } \\
\mathrm{V}=-\dot{\mathrm{V}} \tau_{\mathrm{RS}}
\end{gathered}
$$




\section{Ramsden and Reynolds}

This relation is exploited in single breath studies of lung mechanics. If flow is plotted against expired volume a straight line is obtained, from which $\tau_{R S}$ can be derived. Note, however, that a linear relation between $\mathrm{V}$ and $\dot{\mathrm{V}}$ will only be evident where the lung is homogeneous with a single value for $\tau_{\mathrm{RS}}{ }^{24} 25$

Integration of equation (2) yields

$$
\frac{\mathrm{Vt}}{\mathrm{Vo}_{\mathrm{o}}}=\mathrm{e}^{-\frac{\mathrm{t}}{\mathrm{RC}}}
$$

from which the volume of gas still present in the lungs, $\mathrm{Vt}$, at any time, $\mathrm{t}$, after the onset of expiration can be calculated (Vo is the total volume expired at the end of a complete expiration).

\section{References}

1 Adamson TM, Collins LM, Dehan M, Hawker JM, Reynolds EOR, Strang LB. Mechanical ventilation in newborn infants with respiratory failure. Lancet 1968;ii:227-31.

2 Northway WH Jr, Rosan RC, Porter DY. Pulmonary disease following respiratory therapy of hyaline membrane disease: bronchopulmonary dysplasia. N Engl J Med 1967;276:257-68.

${ }^{3}$ Hawker JM, Reynolds EOR, Taghizadeh A. Pulmonary surface tension and pathological changes in infants dying after respirator treatment for severe hyaline membrane disease. Lancet 1967;ii: $75-7$

4 Taghizadeh A, Reynolds EOR. Pathogenesis of bronchopulmonary dysplasia following hyaline membrane disease. $A m J$ Pathol 1976:82:241-58.

Reynolds EOR. Effect of alterations in mechanical ventilator settings on pulmonary gas exchange in hyaline membrane disease. Arch Dis Child 1971;46:152-9.

${ }^{6}$ Herman S, Reynolds EOR. Methods for improving oxygenation in infants mechanically ventilated for severe hyaline membrane disease. Arch Dis Child 1973;48:612-7.

7 Smith PC, Schach MS, Daily WJR. Mechanical ventilation of newborn infants: II. Effects of independent variation of rate and pressure on arterial oxygenation of infants with respiratory distress syndrome. Anesthesiology 1972:37:498-502.

* Strang LB. Neonatal respiration. Oxford: Blackwell, 1977.

${ }^{9}$ Agostoni E, Taglictti A, Agostoni F. Setnikar I. Mechanical aspects of the first breath. J Appl Physiol 1958:13:344-8.

10 Reynolds EOR. Pressure waveform and ventilator settings for mechanical ventilation in severe hyaline membrane disease. Int Anesthesiol Clin 1974;12:259-80.

1 Reynolds EOR. Management of hyaline membrane discase. $\mathrm{Br}$ Med Bull 1975;31:81-4.

12 Reynolds O. Ventilator therapy. In: Thibeault DW, Gregory GA, eds. Neonatal pulmonary care. Menlo Park: AddisonWesley, 1979:217-36.

13 Reynolds EOR, Taghizadeh A. Improved prognosis of infants mechanically ventilated for hyaline membrane disease. Arch Dis Child 1974;49:505-15.

14 Primhak RA. Factors associated with pulmonary air leak in premature infants receiving mechanical ventilation. $J$ Pediatr 1983;102:764-8

15 Tarnow-Mordi WO, Narang A, Wilkinson AR. Lack of association between barotrauma and air leak in hyaline membrane disease. Arch Dis Child 1985;60:555-9.

16 Bland RD, Kim MH, Light MJ, Woodson JL. High frequency mechanical ventilation in severe hyaline membrane disease. Crit Care Med 1980;5:275-80.

17 Spahr RC, Klein AM, Brown DR, MacDonald HM, Holzman IR. Hyaline membrane disease: a controlled study of inspiratory to expiratory ratio in its management by ventilator. Am J Dis Child 1980;134:373-6.

18 Heicher DA, Kasting DS, Harrod JR. Prospective clinical comparison of two methods for mechanical ventilation of neonates: rapid rate and short inspiratory times versus slow rate and long inspiratory time. J Pediatr 1981;98:957-61.

19 Pohlandt F, Bernsau V, Feilen K-D, et al. Reduction of barotrauma in ventilated neonates by increase in ventilation frequency-first results of a prospective collaborative and randomized trial of two different ventilatory techniques. Pediatr Res 1986;19:1077.

20 Field D, Milner AD, Hopkin IE. High and conventional rates of positive pressure ventilation. Arch Dis Child 1984;59:1151-4.

21 Field D, Milner AD, Hopkin IE. Manipulation of ventilator settings to prevent active expiration against positive pressure inflation. Arch Dis Child 1985;60:1036-40.

22 Greenough A, Morley CJ, Pool J. Are fast rates an effective alternative to paralysis? Pediatr Res 1986;19:1077.

23 Mortola JP. Fisher JT, Smith B, Fox G, Weeks S. Dynamics of breathing in infants. $J$ Appl Physiol 1982;52:1209-15.

24 Thomson A, Silverman M. Single-breath measurement of lung mechanics in very low birthweight infants. Crit Care Med 1985;13:4-8.

25 Le Souef PN, England SJ, Bryan CA. Passive respiratory mechanics in newborns and children. Am Rev Respir Dis 1984:128:552-6.

26 Grunstein MM, Inscore SC. A new method to assess respiratory mechanics in infants with pulmonary disease. Pediatr Res 1986; 20:472.

27 Simbruner G. Inadvertent positive end-expiratory pressure in mechanically ventilated newborn infants: detection and effect on lung mechanics and gas exchange. J Pediatr 1986;108:589-95.

28 Prendiville A, Thomson A. Silverman M. Effect of tracheobronchial suction on respiratory resistance in intubated preterm babies. Arch Dis Child 1986;61:1178-83.

29 LeSouef PN, England SJ, Bryan AC. Total resistance of the respiratory system in preterm infants with and without an endotracheal tube. J Pediatr 1984;104:108-11.

30 Perez Fontan JJ, Heldt GP, Targett RC, Willis MM, Gregory GA. Dynamics of expiration and gas trapping in rabbits during mechanical ventilation at rapid rates. Crit Care Med 1986:14:39-47.

31 Helliesen PJ, Cook CD, Friedlander L, Agathon S. Studies of respiratory physiology in children: 1 . Mechanics of respiration and lung volumes in 85 normal children 5 to 17 years of age. Pediatrics 1958;22:80-93.

32 Olinsky A, Bryan AC, Bryan MH. A simple method of measuring total respiratory system compliance in newborn infants. $S$ Afr Med J 1976;50:128-30.

33 Greenough A, Wood S, Morley CJ, Davis JA. Pancuronium prevents pneumothoraces in ventilated premature babies who actively expire against positive pressure inflation. Lancet 1984; 1-3.

34 Perlman JM, Goodman S, Kreusser KL, Volpe JJ. Reduction in intraventricular hemorrhage by elimination of fluctuating cerebral blood-flow velocity in preterm infants with respiratory distress syndrome. $N$ Engl J Med 1985;312:1353-7.

35 Ramsden CA. Active expiration or synchrony? Arch Dis Child 1986;61:820.

36 Kosch PC, Stark AR. Dynamic maintenance of end-expiratory lung volume in full term infants. J Appl Physiol 1984;57: 1126-33.

37 Vinegar A, Sinnett EE, Leith DE. Dynamic mechanisms determine functional residual capacity in mice, Mus musculus. J Appl Physiol 1979;46:867-71.

${ }^{3 \times}$ Reynolds EOR, Hope PL, Whitehead MD. Muscle relaxation and periventricular hemorrhage. $N$ Engl J Med 1985;313:955-6.

39 Marchak BE, Thompson WK, Duffty P, et al. Treatment of RDS by high-frequency oscillatory ventilation: a preliminary report. J Pediatr 1981;99:287-Y'2.

Correspondence to Dr C A Ramsden Department of Paediatrics, University College London, The Rayne Institute, University Street, London WC1E 6JJ. 